violettes; or, on sait que ce rayonnement intervient fortement dans la fixation du calcium par les êtres vivants : on peut penser, dans ces conditions, que le lait alpin produit par des animaux irradiés est riche à la fois en calcium et en nombreux autres principes bienfaisants. L'influence de l'ensoleillement est encore confirmée par ce fait que les laits d'été sont plus riches en calcium que les laits d'hiver des mêmes régions et que, d'autre part, il ne s'agit pas de l'influence de l'alimentation puisque le lait des vaches enfermées dans des étables ensoleillées (vallée de la Clairée) est plus riche en calcium que celui des vaches dont les étables ne laissent guère pénétrer la lumière du jour (vallée des Aigues).

En résumé, le lait alpin est moins riche en phosphore (caséine, phosphates) que les autres laits français; mais, par contre, il est nettement plus riche en calcium, en lactose et en chlorures. En conséquence, sa constante moléculaire simplifiée et corrigée est plus élevée. Quelle que soit l'influence importante des divers facteurs climatiques sur la composition qualitative et quantitative du lait alpin et sur les variations de sa constante moléculaire simplifiée et corrigée, il apparaît que cette constante chimique est, dans le cadre exclusivement montagnard autant que dans les autres provinces françaises, un test sûr pour le contrôle du lait.

\title{
LA FLUORESCENCE DU BEURRE
}

\section{par \\ MAURICE - DÉRIBÉRÉ}

Dans une précédente étude, nous avons indiqué en ces colonnes les données générales concernant la fluorescence du lait et les indications qui peuvent en être tirées du point de vue du contrôle et des identifications [1].

Le même intérêt se retrouve dans le comportement du beurre sous les rayons ultra-violets filtrés (lumière de Wood).

Lorsqu'on examine une motte de beurre de lait de vache, pur et frais, sous la lumière de Wood, on observe une fluorescence jaune canari, bien nette. En couches très minces cette fluorescence s'atténue, mais subsiste encore.

Toutefois, par irradiation en lumière du jour ou sous les rayons ultra-violets, la fluorescence jaune s'affaiblit et tend à disparaître, aussi devra-t-on faire les essais sur un échantillon prélevé frais à l'intérieur de la motte et non en surface.

De ce fait l'examen en fluorescence permet, par examen de surface, de distinguer un beurre frais d'un beurre rance ancien. La 
fluorescence jaune, en effet, s'atténue peu à peu pour laisser place, au bout de dix à quinze jours, à une fluorescence faible, de teinte crème. Celle-ci disparaît ensuite à son tour, cependant qu'apparaît, au bout de soixante à soixante-dix jours, une fluorescence bleutée assez analogue à celle de diverses graisses animales.

Il faudra cependant remarquer que l'exposition au soleil conduit à une accélération très nette de ces phénomènes tandis que du beurre conservé dans l'obscurité conservera bien plus longuement sa luminescence. Les valeurs que nous avons indiquées ne sont valables qu'en lumière diffuse et la méthode est, par suite d'application, peu étendue et délicate. Elle peut cependant rendre des services.

Par contre, dans la recherche des falsifications par addition de graisses, l'examen par fluorescence est d'un intérêt incontestable.

En effet, les graisses artificielles et animales généralement utilisées pour la fasification des beurres sont obscures ou peu fluorescentes en lumière de Wood; en outre leur fluorescence, lorsqu'elle existe, tend vers le bleu. Tout ceci contraste de façon heureuse avec la fluorescence jaune du beurre frais et permet un contrôle facile et rapide.

\section{Principaux travaux}

De nombreux travaux ont été publiés sur la fluorescence du beurre et nous en avons relevé un certain nombre dans un ouvrage général [2] et dans un précédent article général [3].

Volmar [4], qui a étudié divers mélanges de beurre et de margarine dans son fluoroseope, a établi que, pour 50 à $100 \%$ de margarine, la fluorescence est de teinte indigo et demeure assez sensiblement constante. Par contre, au-dessous de $50 \%$ l'intensité de cette fluorescence diminue progressivement pour disparaître vers $10 \%$. Au-dessous de $10 \%$ l'examen ordinaire simple en lumière de Wood ne permet pas de déceler aisément la présence de margarine dans le beurre à fluorescence jaune, mais l'examen au spectroscope permet encore de constater, jusqu'à quelques $\%$, une faible lueur bleuâtre.

BAUMGARTEL [5] a indiqué que, la margarine avait une fluorescence bleu blanchâtre et que son addition au beurre se distingue plus aisément par fluorescence lorsque l'on dissout les graisses dans l'éther de pétrole. BAKER et TAUBES [6] appliquent l'essai direct de fluorescence à la recherche des fraudes du beurre.

Hokı [7] étudie le mélange de margarine et autres graisses dans le beurre qu'il reconnait à partir de $20 \%$, mais cet auteur étend ses recherches à l'évolution de la fluorescence par vieillissement. Un échantillon de beurre conservé pendant seize jours à la lumière diffuse a donné, sur $3 \mathrm{~mm}$. d'épaisseur, une coloration jaune blan- 
châtre avec légère fluorescence bleue. Un échantillon conservé à l'abri de la lumière montrait une couleur jaune plus foncée, mais se reconnaissait plus facilement à la lumière ordinaire qu'en lumière de Wood. Au bout de soixante-dix jours, l'aspect avait peu changé, mais, par contre, en lumière de Wood, on observait une fluorescence bleutée bien nette.

Le même auteur, en collaboration avec Novacek, a examiné divers mélanges frais dans les couches intérieures de l'échantillon en comparaison avec des échantillons standards de référence. Des teneurs inférieures à $20 \%$ en graisses animales ont pu être ainsi décelées. Il est fait remarquer que le réfractomètre et le micropolarimètre ne peuvent ici servir qu'accessoirement puisque les indices de réfraction et de polarisation du beurre pur se rapprochent souvent de ceux du beurre artificiel. Pour le beurre rafraîchi, pourtant, le micropolarimètre de Litterscheid a donné de meilleurs résultats.

Une importante étude est celle de HaITINGER et REICH qui indiquent [9] que les mélanges sont plus faciles à identifier par mise en solution dans l'éther de pétrole. On distingue de cette façon $10 \%$ de margarine dans le beurre frais.

\section{Résultats et interprétation}

Il ressort de ces divers travaux, qu'il nous a été loisible de vérifier, que l'introduction des graisses usuellement employées en falsification du beurre donne une fluorescence bleutée, lilas ou violacée utilisable dans les contrôles.

Les produits d'addition comportant presque toujours de l'huile de sésame dont la fluorescence est bleue ou lilas, on a été tenté d'attribuer la fluorescence bleutée des beurres falsifiés à cette huile, mais il n'en est rien puisque la margarine, qui ne contient pas d'huile de sésame, montre la même modification.

La graisse de cheval, par contre, et ceci a déjà été signalé par Novacek et HöKL, est assez difficile à différencier. Par contre, les échantillons de matières grasses végétales et particulièrement ceux extraits des noix de coco donnent avec intensité et à partir de 5 à $10 \%$ la fluorescence bleutée.

Ces divers points montrent que, lorsque l'élément en mélange n'est pas connu en nature, l'évaluation quantitative est fort délicate et ne doit être abordée qu'avec prudence.

La fluorescence jaune canari du beurre est due à des complexes dans lesquels la vitamine $B_{2}$, les stérols et divers pigments jouent leur rôle.

L'acide butyrique est peu fluorescent [10]. Il montre seulement, sous forme anhydre, une légère teinte jaune en lumière de Wood [11]. 
Lorsque l'on met le beurre en solution dans l'éther de pétrole, la fluorescence jaune s'atténue jusqu'à disparaitre pratiquement. Par contre, la fluorescence bleue de la margarine et des graisses subsiste et, de ce fait, devient même plus nette. Ceci explique l'intérêt de cet essai par mise en solution préconisé par HAITINGER et par BAUMGARTel et sur lequel nous devons insister ici. Cette forme d'essai devra toujours suivre l'examen direct.

Nous avons aussi obtenu d'intéressants résultats en complétant les deux essais ci-dessus par celui des bandes de capillarité. Une bande fine de papier filtre est trempée par son extrémité dans la solution de la substance, dans l'éther pendant une heure, puis séchée. On examine ensuite cette bande en luminescence. Elle montre, pour le beurre pur une zone jaune de remontée capillaire, tandis que les mélanges se manifestent par des zones alternées jaunes et bleuâtres.

Enfin, l'emploi de filtres permet d'obtenir d'intéressants résultats. Déjà Morcan et MACLennaN [12] a vaient déterminé dans quelques échantillons, au moyen du colorinnètre trichromatique Guild la proportion des trois couleurs fondamentales de l'émission de fluorescence du beurre et de la margarine.

$\begin{array}{lccc} & \text { Rouge } & \text { Vert } & \text { Bleu } \\ \text { Beurre d'Irlande } \ldots \ldots \ldots & 27 & 44 & 29 \\ \text { Beurre du Danemark .... } & 24 & 41 & 35 \\ \text { Margarine I } \ldots \ldots \ldots \ldots \ldots & 10 & 24 & 66 \\ \text { Margarine } 2 \ldots \ldots \ldots \ldots \ldots & 9 & 22 & 69\end{array}$

Ces chiffres sont bien significatifs et rendent compte des faits observés par les divers auteurs précités et de la possibilité des contrôles et identifications. Ils nous montrent aussi que l'emploi de filtres sera intéressant pour l'observation comparative des fluorescences. De fait, en opérant les essais avec un filtre bleu éliminant la fluorescence jaune de fond, nous avons pu obtenir des résultats plus précis que dans l'examen direct ; l'emploi de ce filtre permet d'éviter la mise en solution dans un solvant pour les observations rapides. On devra faire une prise interne dans la motte, la passer en lumière de Wood et l'examiner, d'abord directement, puis à travers le filtre bleu monochromatique (C. 5 Wratten par exemple) ou bleu Signal Corex). En utilisant des gammes-étalons de comparaison, un précontrôle de base aisé et rapide peut ainsi être établi de facon fort simple.

\section{RÉEÉRENCES}

[1] M. Déribéré. La fluorescence du lait. Le Lait, 22, 214-216, avriljuin 1942, p. 122 . 
[2] M. Déribéré. Les applications pratiques de la luminescence, Ed. Dunod, Paris, 1938.

[3] M. Déribéré. L'examen du beurre et de ses falsifications sous la lumière de Wood. Ann. Hygiène Publ., nº 3, mars 1937.

[4] Y. Volmar. L'utilisation des phénomènes de fluorescence dans l'ana1yse des matières alimentaires. Jour. Pharm. Chim, t. V, 1927, p. 435 .

[5] F. Baumgartel. Analyse par luminescence des produits du lait et des fromages. Molkerei Zeit., no 45, 1931, p, 141.

[6] G. W. BaKer et S. TAUBES. Fluorescence du beurre et du lait en lumière ultra-violette. The Analyst, t. LVII, 1932, p. 375.

[7] J. HokL. L'image que donne à la lumière ultra-violette filtrée le beurre rance ou contenant des graisses étrangères. Zeits. Untersuch. Lebensm., t. LXIII, $\mathrm{n}^{\circ} 4$, avril 1932, p. 144.

[8] E. Novack et J. Hokt. Examen de mélanges de diverses graisses à la lumière ultra-violette filtrée de la lampe de quartz de Hanan. Zeits. Fleisch.-u Milchyg., t. XLII, no 3, $1^{\text {er }}$ novembre 1931, p. 47.

[9] M. Haitinger et V. Reich. Sur le comportement de quelques produits de l'agriculture en lumière ultra-violette. Fortschritte der Landwirtschaft, $\mathrm{n}^{\circ} 10,15$ mai $1928, \mathrm{p}, 433$.

[10] A. Nicthammer. L'examen de quelques produits sous la lampe de quartz d'analyse. Zeits. Unters. Lebensmittel, t. LVII, 1929, p. 354.

[11] B. Lustig et G. Botstiber. Sur une méthode de recherche et d'identification des corps gras et acides gras. Biochem.Zeits., t. CCII, 1928, p. 84 .

[12] R. S. Morgan et K. Maclennan. La fluorescence de quelques graisses contenant la vitamine A. Biochem. Jour,, t. XXII, 1928, p. 1514.

\title{
REVUE
}

\section{L'INDUSTRIE LAITIÈRE A L'ÉTRANGER}

\author{
par G. GENIN
}

Ingénieur E. P. C.

\section{Production mondiale du lait}

Pendant les années 1934-1938, la production laitière annuelle européenne a été, en moyenne, de 135.000 millions de kilogrammes. La production nord-américaine serait de 60.000 millions de kilogrammes, dont les $4 / 5^{e}$ seraient produits aux Etats-Unis. La prō. duction sud-américaine serait de 9.000 millions de kilogrammes, l'Argentine et le Brésil en produisant chacun un tiers. La production asiatique, non compris la partie asiatique de la République des Soviets, serait de 40.000 millions de kilogrammes, dont $22.000 \mathrm{mil}$ lions de kilogrammes produits aux Indes et se décomposant en 14.000 millions de kilogrammes de lait de vache et 8.000 millions 